\title{
Extraction of Highly Charged Ions from the Electron Beam Ion Trap at LBNL for Applications in Surface Analysis and Materials Science
}

\author{
T. Schenkel ${ }^{1}$, A. Persaud, A. Kraemer, J. W. McDonald ${ }^{*}$, J. P. Holder ${ }^{*}$, A. V. Hamza*, \\ and D. H. Schneider ${ }^{*}$ \\ E. O. Lawrence Berkeley National Laboratory, Berkeley, CA 94720, USA \\ *Lawrence Livermore National Laboratory, Livermore, CA 94550, USA
}

We describe results from highly ion extraction experiments at the Electron Beam Ion Trap (EBIT) facility which is now operated at Lawrence Berkeley National Laboratory after transfer from Lawrence Livermore National Laboratory. Requirements on ion source performance for the application of highly charged ions (e. g. $\left.\mathrm{Xe}^{44+}\right)$ in surface analysis and materials science are discussed.

\section{Introduction}

The Electron Beam Ion Traps (EBIT) at Lawrence Livermore National Laboratory were originally developed for x-ray spectroscopy and fundamental atomic physic studies [1]. Over ten years ago, extraction of beams of highly charged, high $\mathrm{Z}$ ions with kinetic energies of a few $\mathrm{keV} / \mathrm{u}$ established the EBIT also as a source for bright beams of slow, highly charged ions $[2,3]$. EBIT II was recently transferred from LLNL to LBNL, and is now operating with extraction potentials and electron beam energies up to $30 \mathrm{keV}$. This ionization energy is sufficient for the efficient formation of any neon like ion, He-like Xe and bare ions up to about $\mathrm{Kr}^{36+}$. The research program at LBNL is focused on the following three areas. First, fundamental ion-solid interaction studies in a regime where 
the interaction is dominated by the deposition of tens to hundreds of $\mathrm{keV}$ of potential energy and the influence of the kinetic energy of projectiles is secondary $[4,5]$. This includes the relaxation dynamics of slow, highly charged ions (HCI) via the formation and decay of super-excited, or hollow atoms. The second area concerns the development of highly charged ions based surface analysis techniques, such as time-of-flight secondary ion mass spectrometry (HCI-SIMS) and emission microscopy $[4,6,7]$. The final area concerns the ongoing development of a single ion implantation facility for the formation of non-Poissonian implant profiles. We briefly review the beam requirements needed to pursue surface analysis and materials science applications.

\section{Extraction of highly charged ions from EBIT II}

In Figure 1, we show a magnet scan with $\mathrm{Xe}^{\mathrm{q}+}$ series extracted from EBIT II. The source was operated in pulsed mode, were the middle drift tube potential is raised above the top drift tube potential after an ion trapping time of $0.5 \mathrm{~s}$. The extraction potential was $7 \mathrm{kV}$, the ionization energy $12 \mathrm{keV}$. Isotope purified ${ }^{136} \mathrm{Xe}$ gas was continuously injected into the trap volume. Trap parameters were selected for the optimization of $\mathrm{Xe}^{44+}$ (neon-like). The ionization energy of $12 \mathrm{keV}$ is also sufficient for the formation of some ions with vacancies in the L-shell. $\mathrm{Xe}^{44+}$ has been the most widely used projectile for surface analysis studies due to the ease of source operation for the formation of adequate probe beams, and because strong electronic sputtering and desorption effects are observed only for charge states larger than about 30+ for Xe [4].

Focusing of the probe beam would allow microanalysis of solid targets without use of an emission microscope scheme [6], or it would improve the sampling speed and

\footnotetext{
${ }^{1}$ Email: T_Schenkel@LBL.gov
} 
probably also the achievable lateral resolution in the $\mathrm{HCI}$ emission microscope. When an ion beam is focused, an object slit or virtual ion source are imaged through a set of lenses with a given demagnification. In liquid metal ion guns, the ions originate from an almost atomically small metal tip, and with $<1 \mathrm{eV}$ energy spread. Hence, spot sizes of 5 to 10 $\mathrm{nm}$ with $\mathrm{Ga}^{+}$currents of over $1 \mathrm{pA}$ are routinely achievable. In an EBIT the ions originate from the electron beam, which has a diameter of about $50 \mu \mathrm{m}$ [8]. Results from energy spread measurements of $\mathrm{Xe}^{44+}$ ions with a reverse field method are shown in Fig. 2. The figure shows the number of detected $\mathrm{Xe}^{44+}$ ions as a function of reverse target bias (open) and the result of numerical differentiation thereof (solid). The extraction voltage of the Xe ions was $7.0 \mathrm{kV}$. The source was operated in leaky mode, so that the ionization energy was also $7 \mathrm{keV}$. The mean energy reduction below the nominal extraction potential by $182 \mathrm{~V}$ results from a space charge neutralization effect by the electron beam during ion extraction [9]. The width of the profile is $15 \mathrm{~V}$, and this value is likely not to limit the achievable spot size through chromatic aberrations. Efforts to focus HCI beams have so far been few. Marrs et al., reported the transport of $\sim 10^{6}$ mostly $\mathrm{Ar}^{18+}$ ions into a spot with a diameter of $20 \mu \mathrm{m}$ [8]. We are aiming at improving this result and have achieved so far a rate of $\sim 10^{3} \mathrm{P}^{12+}$ ions transported through a $5 \mu \mathrm{m}$ aperture (Figure 3 a). Here, a magnet scan of extracted ions shows the $\mathrm{P}^{\mathrm{q}+}$ series in leaky mode. The extraction potential, $8.5 \mathrm{kV}$, was equal to the ionization potential. Beam profiles measurements are in progress. Phosphorous was introduced into the source through a gas injector as $\mathrm{PF}_{3}$. In leaky mode, the trap is not pulsed, but the beam is formed through continuous evaporation of ions over the trap barrier. The trap had a depth of $100 \mathrm{~V}$. The instantaneous ion rate in leaky mode is smaller than in pulsed mode, but without the 
limitation of a trap cycle rate of only one to a few $\mathrm{Hz}$ in pulsed mode higher ion extraction rates per second can be achieved. A magnet scan with $\mathrm{P}^{\mathrm{q}+}$ series in pulsed mode is shown in Fig. 3 b. Here, the ionization potential was $8 \mathrm{keV}$, but the extraction potential was $5.8 \mathrm{kV}$. In this plot the magnetic field scale was projected onto a $\mathrm{m} / \mathrm{q}$ scale to underline the separation of $\mathrm{m} / \mathrm{q}=2$ ions (fully stripped light ions), from m/q=2.067 for ${ }^{31} \mathrm{P}^{15+}$, and $\mathrm{m} / \mathrm{p}=2.11$ for ${ }^{19} \mathrm{~F}^{9+}$. The origin of the small shoulders at higher $\mathrm{m} / \mathrm{q}$ for the main $\mathrm{P}^{\mathrm{q}+}$ peaks is currently under investigation. Leaky mode operation shifts the charge state distribution to lower q. The reason for that is the higher trap potential for higher ion charge states, and increased charge exchange with lighter ions in the trap. Charge exchange is a problem when using $\mathrm{PF}_{3}$ since a parasitic light ion species is introduced with three times higher abundance then the desired $\mathrm{P}$. In pulsed mode, bare $\mathrm{P}$ ions are readily obtained as the most abundant species.

\section{Beam requirements for applications}

\subsection{Beam requirements for surface analysis with highly charged ions}

The analysis of the chemical composition and elemental structure of surfaces with ever increasing spatial resolution and sensitivity is important for many areas of applied science, including semiconductor processing. In the latter the conditions of silicon wafers have to be controlled with great finesse. In the array of surface analysis techniques available today [10], time-of-flight secondary ion mass spectroscopy with singly charged projectiles offers a sensitivity of $\sim 10^{8}$ atoms $/ \mathrm{cm}^{2}$ for most transition metals on oxidized silicon, parallel mass detection which includes molecular ions and a lateral resolution as low as $100 \mathrm{~nm}$ [11]. The finding of very high yields of atomic and 
molecular secondary ions that are emitted from oxidized silicon surfaces following the impact of slow (few Kev/u) highly charged ions (e. g. Xe ${ }^{44+}$ ) motivated the development of a time-of-flight secondary ion mass spectrometry scheme with HCI [3]. One central idea in HCI-SIMS is that the start of each TOF cycle is triggered by one incident ion. In negative (positive) polarity, secondary electrons (protons) are detected and start the TOFcycle, and the arrival times of the trail of heavier secondary ions allow m/q identification in a linear or reflectron type TOF spectrometer. In conventional TOF-SIMS, the primary ion beam is pulsed with pulse widths of several hundred ps. The beam from an EBIT can also be pulsed, but achievable pulse lengths are prohibitively long for TOF-SIMS adaptations. TOF-cycle times depend on the ion flight path, about $1 \mathrm{~m}$ in a reflectron, and on the extraction potential. The latter is about $5 \mathrm{kV}$ and resulting flight times for heavy ( 1000 Da) secondary ions are a few hundred $\mu$ s. The start pulse from the $\mathrm{n}^{\text {th }}$ ion can also be used to switch a voltage on a steering element in the beam line so as to prevent the $\mathrm{n}+1$ ion to strike the target before the TOF cycle of the $\mathrm{n}^{\text {th }}$ ion is completed. Such events increase the background signal and have to be avoided. Minimization of this background source gives a criterion for the number of HCI that should impinge on a target per second. In the interest of short analysis times, this number should be large, and we get a rate of $10^{4}$ ions $/ \mathrm{s}$ for a $0.1 \mathrm{~ms}$ TOF-cycle. Since ions arrive randomly, we can reduce the background from accidental coincidences that escape the switch off with a TOF-cycle rate of about $1 \mathrm{kHz}$. If each $\mathrm{HCI}$ desorbs secondary ions from an estimated area of $100 \mathrm{~nm}^{2}$, then an area of $10 \mu \mathrm{m} \times 10 \mu \mathrm{m}$ will be interrogated within about $15 \mathrm{~min}$. of analysis time. This analysis will cover the static regime, where the top few 
monolayers of a target are of concern. Measuring longer than that yields information of deeper sample layers that have already been exposed to and altered by the probe beam.

This example shows that an intensity of a few $1000 \mathrm{HCI} / \mathrm{s}$ suffices for this application. Typical intensities of $\mathrm{Xe}^{44+}$ ions from EBIT II are of order $10^{6}$ ions/s for the full beam. Use of electrostatic lenses for focusing and collimation with beam limiting apertures allows formation of micron size beams (see above). Focusing of higher numbers of ions/s into smaller spot sizes would allow access to the dynamic analysis regime (e. g. for depth profiling of dopants in silicon). Tradeoffs between the three parameters of ion impact rate, spot size and analysis time can be made for specific applications. E. g., a sample with a homogeneous coverage of trace elements on the top surface can be analyzed for hours with a low ion rate to accumulate statistics over a larger beam spot.

\section{2. Materials modification with highly charged ions}

The most widely used technique for materials modification with ion beams is ion implantation. State of the art implanters deliver broad, space charge limited beams of mostly $\mathrm{B}^{1+}$ and $\mathrm{P}^{1+}$ ions with keV energies to silicon wafers and implant large areas within seconds. Clearly, there is no point in pursuing such a thing with an EBIT, since the achievable ion beams lack about 8 orders of magnitude in intensity. Materials modification with highly charged ions from an EBIT is aimed at effects of single ion impacts with very high charge states (e. g. $>40+$ for $\mathrm{Xe}$, and $>60+$ for Au). The high ion charge is important to access a regime of intense, ultra fast electronic excitations $[3,12]$, where the deposition of, e. g. $170 \mathrm{keV}$ of potential energy of a $\mathrm{Au}^{69+}$ ion within 5 to $10 \mathrm{fs}$ 
and in an area of $\sim 10 \mathrm{~nm}^{2}$ results in a power density at the surface of $\sim 10^{13} \mathrm{~W} / \mathrm{cm}^{2}$. The value for the size of the primary area of energy deposition is chosen somewhat arbitrarily. Electron emission is localized even more, while single ion defects on self assembled monolayers where formed to extend over $50 \mathrm{~nm}$ [4]. Since the latter is a result of energy dissipation following primary relaxation processes, we side with the shorter length scale in the assessment of the initial power density. Terra watt power densities can also achieved with ultra short pulse lasers, but the laser beam spots are of $\mu \mathrm{m}$ extensions. Hot electrons travel $\mathrm{nm}$ in fs, so that they can leave the nanometer scale areas of high excitation density from HCI impacts before they thermalize down and transfer their excess energy to the lattice. The initial effect of the intense fs excitation is thus "frozen in" on the nanometer scale for HCI, while the fs effects from short pulse lasers are superimposed with conventional melting on a ps time scale.

Several single ion impact effects of HCI, such as blisters on mica [3], craters of self assembled monolayers [4], and recently light emitting silicon structures [13], were discovered in studies with unfocused beams. Here, HCI impinge on samples randomly and cover an area of several $\mathrm{mm}^{2}$ with a low dose of $\sim 10^{9} \mathrm{HCI} / \mathrm{mm}^{2}$. At a beam current of $10^{5}$ to $10^{6} \mathrm{HCI} / \mathrm{s}$, such exposures can be completed in a few hours.

The motivation for the formation of focused beams of highly charged phosphorous ions is single ion implantation of individual $\mathrm{P}$ ions into nanometer scale areas for the formation of electronic devices functioning with small numbers of dopant atoms. Details of our scheme for the formation of non-Poissonian implant structures with $\mathrm{P}^{15+}$ ions will be described in an upcoming publication [14]. 


\section{Conclusion}

EBIT II is a well optimized, reliable source for slow (few keV/u), highly charged ions and the routinely achievable beam currents of up to $10^{6}$ ions/s are well suited for surface science applications of HCI in a time-of-flight secondary ion mass spectrometry scheme and for basic and applied studies in materials modification. A program for beam focusing is underway and transport of $10^{3} \mathrm{P}^{12+}$ ions through a $5 \mu \mathrm{m}$ aperture has been achieved.

\section{Acknowledgements}

The This work is supported by the Director, Office of Science, Office of Basic Energy Sciences, of the U.S. Department of Energy under Contract No. DE-AC0376SF00098, and by the National Security Agency (NSA) and Advanced Research and Development Activity (ARDA) under Army Research Office (ARO) contract number MOD707501. Work at LLNL was performed under the auspices of the U. S. Department of Energy by Lawrence Livermore National Laboratory under contract No. W-7405ENG-48. A. K. was supported by the Deutsche Akademische Austauschdienst (DAAD).

\section{References}

[1] M. A. Levine, R. E. Marrs, J. R. Henderson, D. A. Knapp ,and M. B. Schneider, Phys. Scr. T22, 157 (1988)

[2] D. Schneider, M. W. Clark, B. M. Penetrante, J. McDonald, D. DeWitt, and J. N. Bardsley, Phys. Rev. A 44, 3119 (1991)

[3] D. H. Schneider, and M. A. Briere, Phys. Scr. 53, 228 (1996) 
[4] T. Schenkel, A. V. Hamza, A. V. Barnes, and D. H. Schneider, Prog. Surf. Sci. 61, 23 (1999)

[5] H. P. Winter, and F. Aumayr, Phys. Scr. T92, 15 (2001)

[6] A. V. Hamza et al., Rev. Sci. Instr. Rev. Sci. Instr. 71, 2077 (2000)

[7] T. Schenkel, et al., Nucl . Instr. and Meth. B 161-163, 65 (1999)

[8] R. E. Marrs, D. H. Schneider, and J. W. McDonald, Rev. Sci. Instr. 69, 204 (1998)

[9] H. Kurz et al., Phys. Rev. A 49, 4693 (1994)

[10] C. R. Brundle, C. A. Evans, and S. Wilson, Encyclopedia of Materials Characterization (Butterworth-Heineman, Stoneham, 1992)

[11] A. Benninghoven, Surf. Sci. 299/300, 246 (1994)

[12] T. Schenkel et al., Phys. Rev. Lett. 81, 2590 (1998)

[13] A. V. Hamza, in press

[14] T. Schenkel, et al., to be published 
Figure captions:

Figure 1: Magnet scan with $\mathrm{Xe}^{\mathrm{q}+}$ series in pulsed mode with a trapping time of $0.5 \mathrm{~s}$. The ionization energy was $12 \mathrm{keV}$, the extraction potential was $7 \mathrm{kV}$.

Figure 2: Number of detected $\mathrm{Xe}^{44+}$ ions as a function of reverse target bias (open) and derivative thereof (solid). The extraction voltage of the Xe ions was $7.0 \mathrm{kV}$. The source was operated in leaky mode.

Figure 3: a) Magnet scan of extracted ions with $\mathrm{P}^{\mathrm{q}+}$ series in leaky mode. The extraction potential, $8.5 \mathrm{kV}$, was equal to the ionization potential. The beam was detected following focusing and collimation through a $5 \mu \mathrm{m}$ aperture. $\mathrm{P}$ is introduced into the trap through gas injection of $\mathrm{PF}_{3}$.

Figure 3: b) Magnet scan with $\mathrm{P}^{\mathrm{q}+}$ series in pulsed mode. The trapping time was $0.8 \mathrm{~s}$ and the ionization potential was $8 \mathrm{keV}$. The extraction potential was $5.8 \mathrm{kV}$. We translated the magnetic field values into an $\mathrm{m} / \mathrm{q}$ scale. 


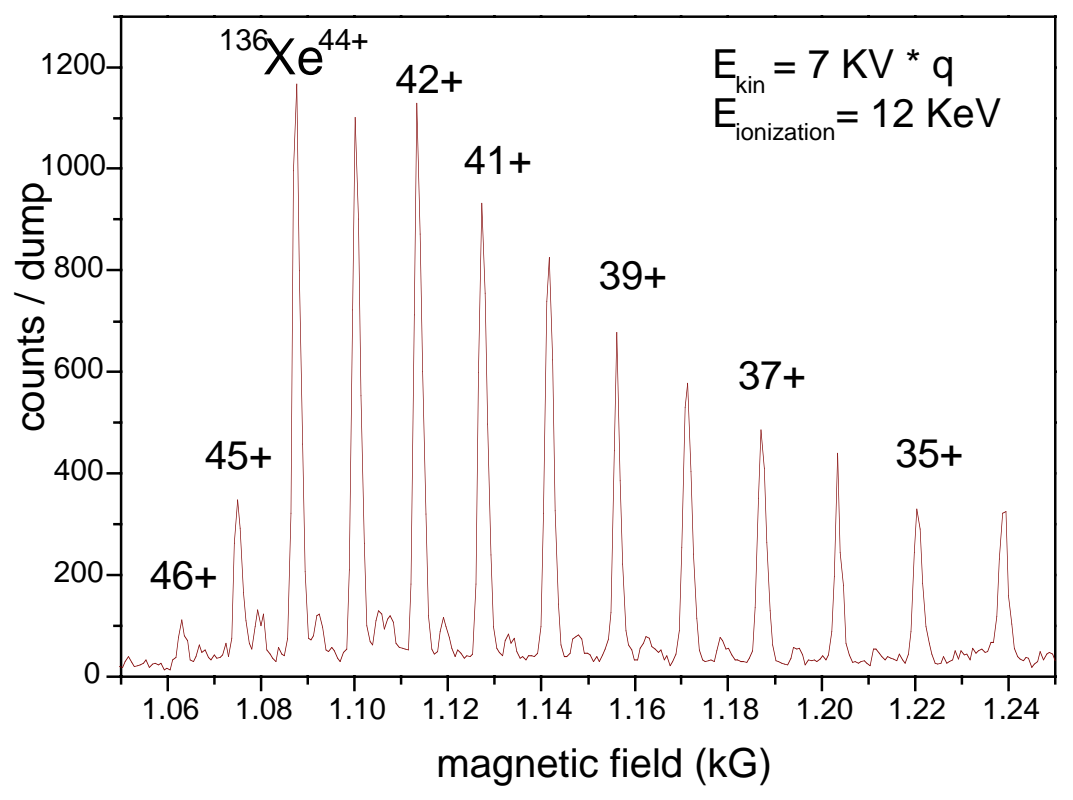




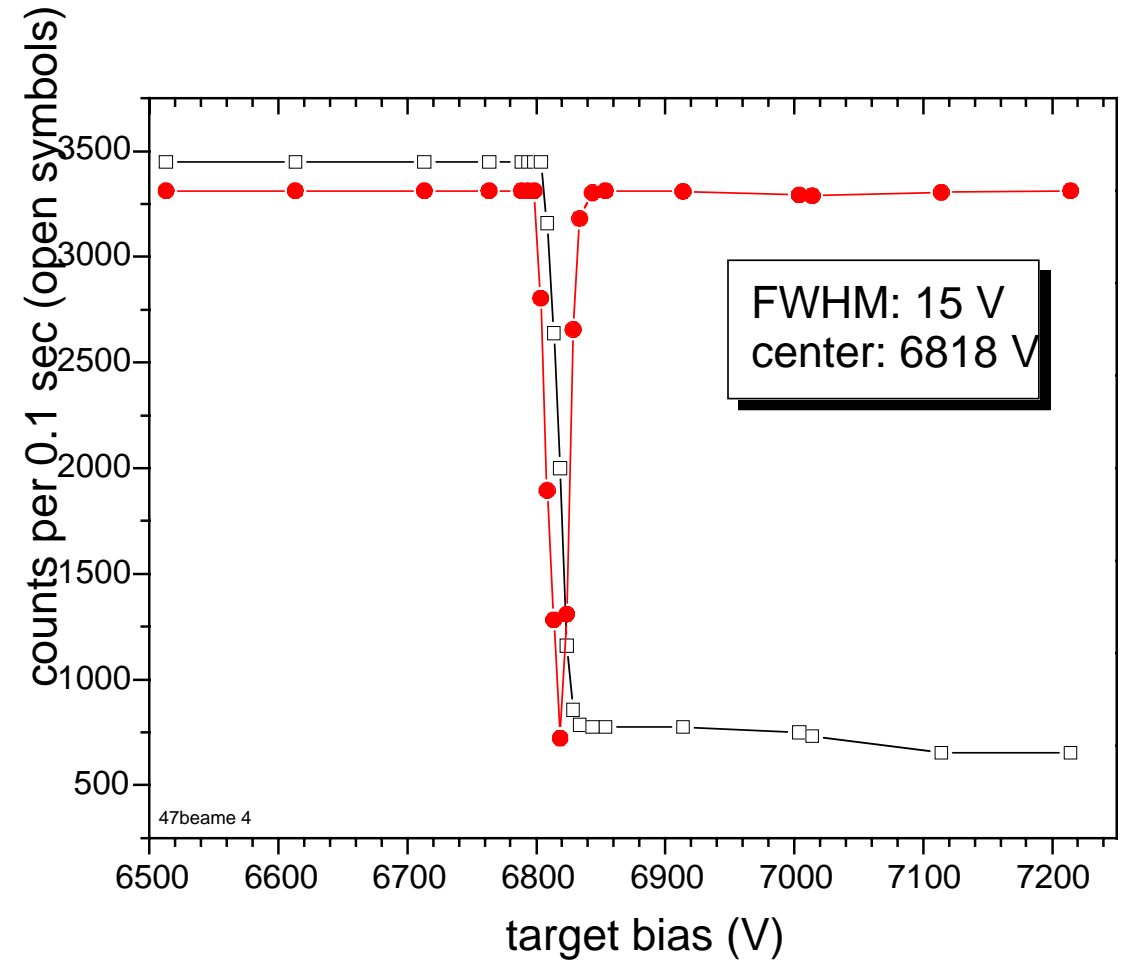




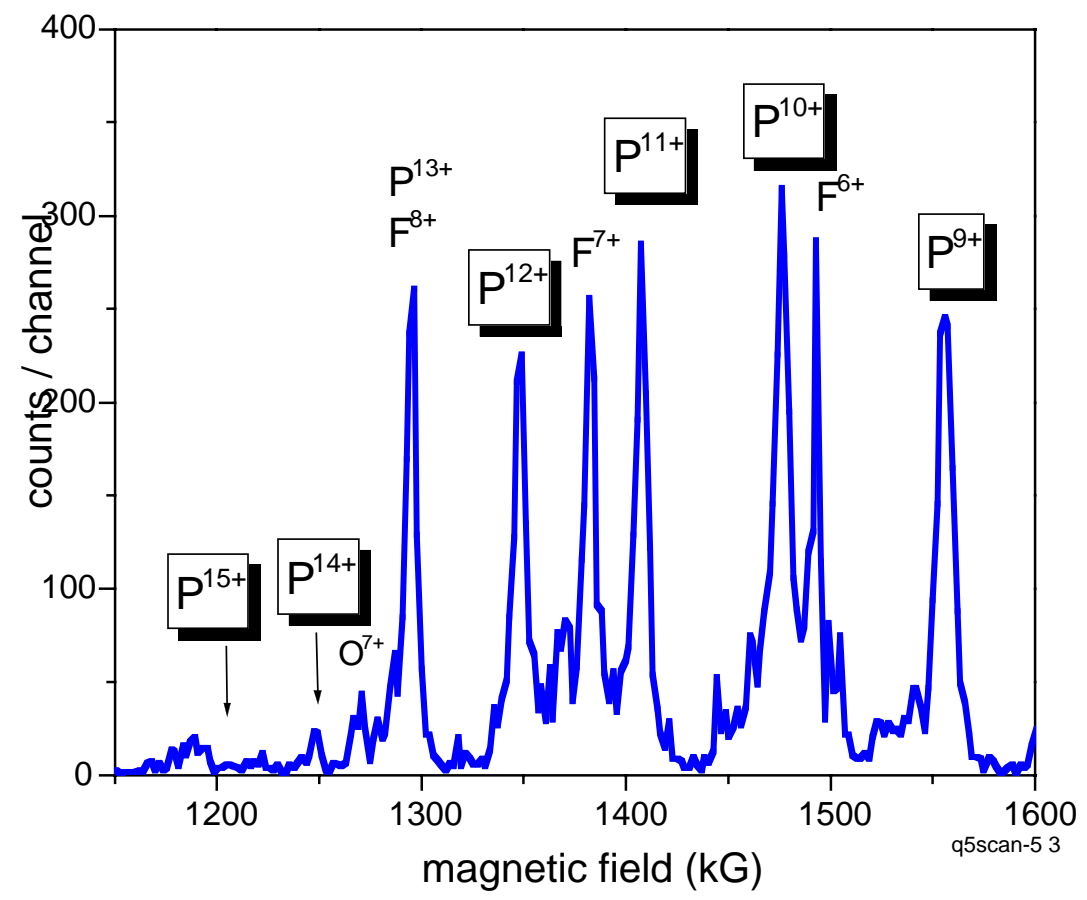




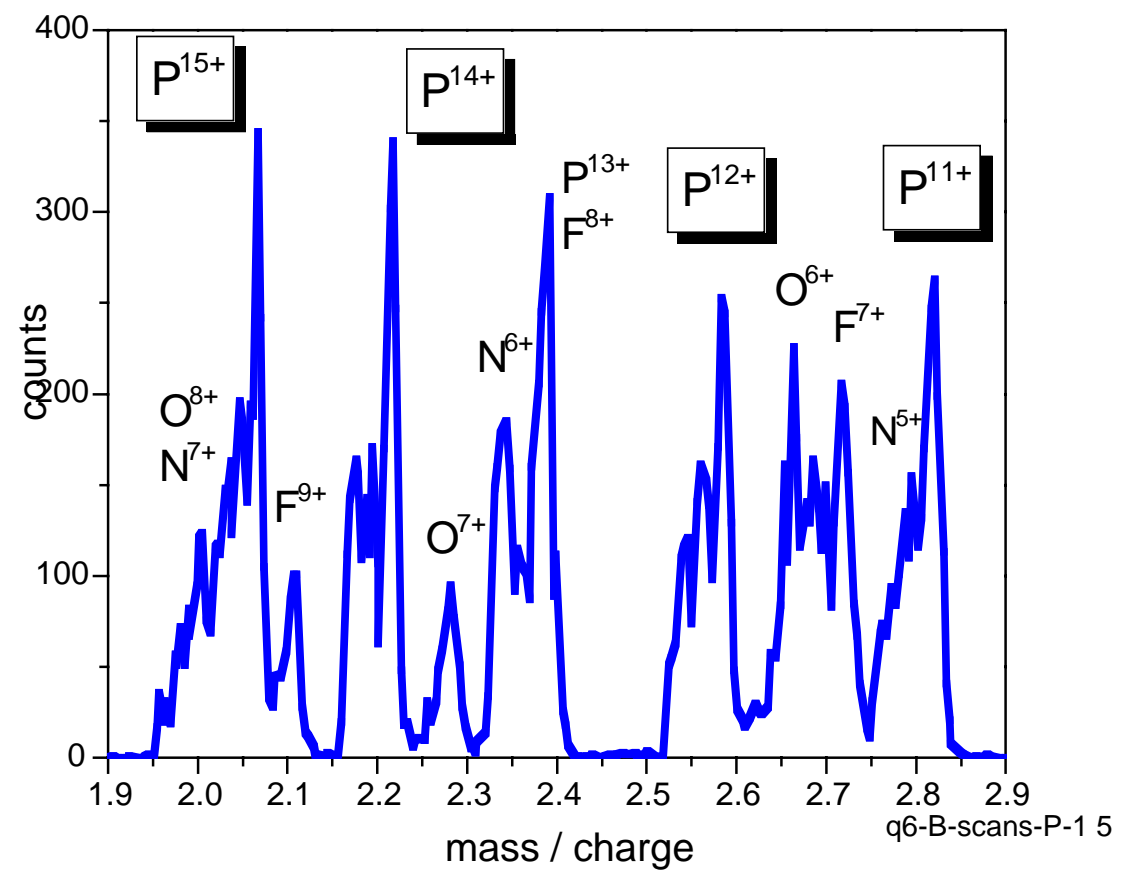

